Number of pages: 16

Number of references: 18

Number of Figures: 9

Number of Tables: 2

\title{
Effect of reclining a seat on the discomfort from vibration and shock
} on fast boats

\author{
Henrietta V.C. Howarth and Michael J. Griffin \\ Human Factors Research Unit, \\ Institute of Sound and Vibration Research, \\ University of Southampton, \\ Southampton, UK \\ SO17 1BJ \\ Tel: $+44(0) 2380592277$
}

Corresponding author.Email: h.howarth@soton.ac.uk 


\begin{abstract}
Passengers and crew on fast boats can experience high magnitudes of whole-body vibration and mechanical shocks that may present risks to health and cause discomfort. This study investigated the influence of reclining a seat on the discomfort caused by fast-boat motion and whether discomfort can be predicted by overall ride values according to current standards. Subjects judged the discomfort of simulations of a recorded fast boat motion in a seat reclined by $0,15,30,45$, or 60 degrees. Reclining the seat caused no significant change in overall discomfort, suggesting that if a reclined seat can be shown to reduce risks of injury it may be acceptable in respect of comfort. The findings are inconsistent with the predictions of standards and show that revised frequency weightings are required to account for seat pan or seat back inclination.
\end{abstract}

Keywords: whole-body vibration; shock; discomfort; boats; reclined seats

\title{
Practitioner Summary
}

Contrary to predictions of current standards, reclining a seat may not increase discomfort during fast-boat motion. Revised frequency weightings for evaluating the severity of whole-body vibration are required to account for seat pan or seat back inclination. 


\section{Introduction}

Crew and passengers on high speed marine craft can be exposed to high magnitudes of whole-body vibration and severe mechanical shocks that may present risks to health, cause discomfort, and affect task performance (ISO 2631-1:1997; ISO 2631-5:2004). Human responses to the vibration and shock can be expected to depend on the biodynamic responses of the body, which are influenced by sitting posture (Messenger and Griffin 1990; Paddan and Griffin 1998; Kitazaki and Griffin 1998; Mansfield and Griffin 2002; Wang et al. 2004).

Supporting the back with a backrest changes the response of the body and may either increase or decrease vibration severity, depending on the frequency and direction of the vibration and the inclination of the backrest (Basri and Griffin 2012, 2013). An upright backrest tends to stiffen the body: the resonance frequencies in the vertical apparent mass measured at the seat surface increased from about $5 \mathrm{~Hz}$ when sitting without a backrest to $5.5 \mathrm{~Hz}$ with a rigid upright backrest (Toward and Griffin 2009). The backrest therefore tends to increase the apparent mass at frequencies greater than the principal resonance frequency and reduce the apparent mass at frequencies less than the principal resonance. In addition to changing the apparent mass, a backrest may increase the transmission of fore-and-aft and pitch vibration to the head (Paddan and Griffin 1988).

As a seat is reclined, more of the weight of the upper-body is supported by the backrest, and static forces in the lower spine (due to the weight of the upper-body) and dynamic forces in the lower spine (due to acceleration from vertical vibration and shocks) can be expected to be reduced. If increased forces in the lower spine are associated with increased risk of injury, it can be hypothesised that the risk of such injuries in high- 
speed craft will be reduced by suitable inclination of seats. However, reclining a seat will increase the transmission of vibration to the upper-back and may increase discomfort. For subjects sitting on a horizontal seat pan with a backrest reclined to 30 degrees or more, there is a trend for greater discomfort at the head or neck during vertical vibration at frequencies greater than $8 \mathrm{~Hz}$ but less discomfort at $5 \mathrm{~Hz}$ and 6.3 Hz (Basri and Griffin 2013). Although there is evidence that reclining a backrest may either increase or decrease the discomfort caused by vertical vibration depending on the frequency of oscillation (Basri and Griffin 2012, 2013; Paddan et al. 2012), there are no known studies with reclined seats and motions similar to those in high-speed craft.

For predicting vibration discomfort, British Standard 6841:1987 and International Standard 2631-1:1997 define frequency weightings and axis multiplying factors for translational and rotational acceleration at the seat, the back, and the feet of a seated person (Table 1). The weightings and multiplying factors were derived from studies with healthy adults. Both standards indicate how frequency-weighted acceleration can be used to quantify vibration severity and suggest the use of the vibration dose value, VDV, when there are significant shocks:

$$
\text { vibration dose value (VDV) }=\left[\int_{t=0}^{t=T} a_{w}{ }^{4}(t) d t\right]^{1 / 4}
$$

where $a_{w}(t)=$ frequency-weighted acceleration.

To combine the vibration in the twelve axes, an 'overall ride value' may be determined by root-sums-of-squares summation of the r.m.s. accelerations, or by fourth-root summation of the r.m.q. accelerations (i.e., root-sums-of-quads summation). Weighted values determined in any axis that are less than $25 \%$ of the maximum value at the same 
point but in another axis may be excluded in the root-sums-of-squares summation. It is stated that where a person is seated on an inclined seat, the relevant orientation should be determined by the axes of the body and therefore the $z$-axis may not be vertical. When a backrest is inclined, vertical vibration of the surface supporting the seat will produce a component in the $x$-axis at the back, so there is both $x$-axis and $z$-axis vibration of the backrest during $z$-axis excitation. Neither British Standard 6841:1987 nor International Standard 2631-1:1997 indicate any adjustment to the evaluation method to allow for the characteristics of a backrest (e.g., height, shape, or inclination). To predict the risks to health, BS 6841 (1987) advocates the use of the vibration dose value calculated from the vibration summed over all three translational directions on the seat and fore-and-aft vibration at the backrest. International Standard 2631-1 (1997) advocates the use of the r.m.s. acceleration or the VDV calculated solely from the acceleration in the direction on the seat surface (not the backrest) that gives the greatest magnitude.

The effects of seat inclination on the discomfort and health of seated persons exposed to vertical whole-body vibration might be predicted from the guidance in BS 6841:1987 by resolving vertical acceleration, $a$, into the $x$-axis and $z$-axis components of a basicentric coordinate systems at the seat and the back (i.e., $a \cdot \sin \theta$ and $a \cdot \cos \theta$, respectively, where $\theta$ is the inclination in degrees) (Howarth and Griffin 2012). With $90^{\circ}$ between the backrest and the seat pan, and a vertical acceleration of $10 \mathrm{~ms}^{-2}$ r.m.s. at frequencies from 0.5 to $16 \mathrm{~Hz}$, overall ride values were calculated for seat pan and backrest inclinations in the range $0^{\circ}$ (upright) to $90^{\circ}$ (fully reclined). Reclining a seat increases the $x$-axis component of acceleration at the seat pan and the seat back but decreases the $z$-axis component of acceleration at both locations. The different frequency weightings for the two locations and both axes of vibration results in seat 
inclination increasing or decreasing the overall ride value depending on the frequency of vibration. At frequencies less than $4 \mathrm{~Hz}$, the overall ride value increases as the seat is reclined, whereas at frequencies greater than $4 \mathrm{~Hz}$, the overall ride value decreases as the seat is reclined (Figure 1). This suggests that reclining a seat will increase discomfort if the vibration is entirely at frequencies less than $4 \mathrm{~Hz}$ but reduce discomfort if the vibration is only at frequencies greater than $4 \mathrm{~Hz}$.

The oscillatory motions on high-speed marine craft can be predominately in the vertical direction (i.e., $z$-axis of a body sitting upright) with the greatest energy in acceleration power spectra around 1 to $2 \mathrm{~Hz}$, but with shocks containing energy at higher frequencies that may contribute to the severity of the motion. Since high-speed craft have motions at frequencies around $1 \mathrm{~Hz}$ and at higher frequencies, it is not obvious whether reclining a seat on a high-speed craft will increase or decrease discomfort.

\section{Table 1 about here}

Figure 1 about here

This study was designed to investigate the influence of reclining a seat on the discomfort of people exposed to a laboratory simulation of motion recorded on a highspeed marine craft. It was hypothesised that discomfort would increase if the severity of the overall ride values, or overall vibration dose values, determined in accord with BS 6841 (1987) increased.

\section{Method}

\subsection{Motion stimuli}

Subjects experienced 20-s reproductions of vertical, fore-and-aft, and pitch motion recorded on a high-speed marine craft and reproduced with high fidelity at frequencies 
in the range 0.4 to $20 \mathrm{~Hz}$ (Figure 2) at six magnitudes. The vertical $W_{\mathrm{b}}\left(\right.$ or $W_{\mathrm{k}}$ ) frequency-weighted acceleration recorded on the simulator platform ranged from 0.26 (0.29) to $0.92(1.05) \mathrm{ms}^{-2}$ r.m.s., with peak acceleration from 1.65 (1.70) to 5.21 (5.34) $\mathrm{ms}^{-2}$. The vertical $W_{\mathrm{b}}\left(\right.$ or $W_{\mathrm{k}}$ ) frequency-weighted vibration dose values for the six 20-s motions ranged from $1.05(1.11)$ to $3.53(3.82) \mathrm{ms}^{-1.75}$, with fore-and-aft $W_{\mathrm{d} \text {-weighted }}$

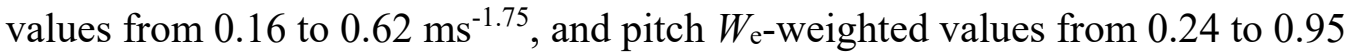
$\operatorname{rads}^{-1.75}$ (Table 2). The motion was therefore predominately vertical.

\section{Figure 2 about here}

\section{Table 2 about here}

\subsection{Seating}

Subjects sat on a rigid aluminium-framed seat attached to the platform of a 6-axis simulator. The seat comprised a flat wooden seat pan and backrest, an adjustable wooden footrest, and a foam-covered headrest. The angle between the seat pan and the backrest, and the knee angle, were fixed at $105^{\circ}$ and $135^{\circ}$, respectively. The inclination of the entire seat (i.e., seat pan, backrest, and footrest) was adjustable by rotation about a pivot point at the junction between the seat pan and the backrest.

Subjects attended one session with six seating conditions (Figure 3):

(i) upright, horizontal seat pan, no backrest or headrest;

(ii) $15^{\circ}$ reclined seat, no headrest;

(iii) $30^{\circ}$ reclined seat, no headrest;

(iv) $30^{\circ}$ reclined seat with headrest;

(v) $45^{\circ}$ reclined seat with headrest;

(vi) $60^{\circ}$ reclined seat with headrest. 
The position of the footrest was adjusted for each subject so that the thighs rested lightly on the seat pan. The subjects were asked to maintain comfortable postures, with their hands on their laps and their feet flat on the footrest. Subjects wore a loose-fitting 5point harness for safety. During conditions (i), (ii) and (iii), subjects were instructed to avoid contact with the headrest and the experimenter observed their posture to confirm that there was no contact with the headrest. During condition (i), subjects were also instructed to avoid contact with the backrest, and the experimenter checked to make sure there was no contact.

\section{Figure 3 about here}

\subsection{Procedure}

In each of the six seating conditions, subjects were presented with the six magnitudes of motion in a balanced random order, resulting in 36 vibration exposures. The six sitting postures were presented in a balanced Latin-square order across subjects.

Judgements of discomfort were obtained for each motion by the method of absolute magnitude estimation (Stevens 1975; Huang and Griffin 2014): subjects were asked to give any numerical values they wished to quantify their relative discomfort. Before the start of the experiment, subjects completed a practice session, first assigning numbers to indicate the relative lengths of lines, and then to indicate the discomfort of 12 practice motions. Subjects also indicated the area of the body in which they experienced most discomfort by providing a number according to areas drawn on a body map.

Subjects wore headphones through which white noise was presented at $70 \mathrm{~dB}(\mathrm{~A})$ in order to mask any sounds of the simulator. The experimenter communicated with subjects through a microphone connected to the headphones by interrupting the white noise. During motion, subjects kept their eyes closed. 


\subsection{Subjects}

Eighteen male subjects aged 18 to 45 years (median $=26$, inter-quartile range, IQR $=8$ ) participated in the study. The median (IQR) body weight and stature of subjects were 72 (21) $\mathrm{kg}$ and $1.75(0.11) \mathrm{m}$, respectively.

Subjects were students or staff of the University of Southampton with no history of any serious illness, injury, or disability. The experiment was approved by the Ethics Committee of the Faculty of Engineering and the Environment at the University of Southampton. All subjects gave informed consent prior to the start of the experiment.

\section{Results}

The magnitude estimates of discomfort given by each subject were normalised such that a value of 100 corresponded to the discomfort experienced with the fourth highest magnitude of vibration $\left(0.52 \mathrm{~ms}^{-2}\right.$ r.m.s. $)$ with the seat upright with no backrest.

\subsection{Rate of growth of discomfort}

With each seat inclination, the median normalised discomfort ratings increased with increasing acceleration (Figure 4).

Figure 4 about here

Magnitude estimates of vibration discomfort, $\psi$, caused by the vibration magnitude, $\varphi$, were assumed to be related by Stevens' power law (Stevens 1975):

$$
\psi=k \varphi^{n}
$$

So,

$$
\log _{10} \psi=n \log _{10} \varphi+\log _{10} k
$$

Individual values for the rate of growth of discomfort, $n$, and the constant, $k$, were determined from the slopes and intercepts of linear least squares regressions between 
$\log _{10} \psi$ and $\log _{10} \varphi$ within each of the six seating conditions. The median rate of growth of discomfort, $n$, ranged across the six seating conditions from $0.627\left(30^{\circ}\right.$ inclined seat, no headrest) to 0.815 ( $60^{\circ}$ inclined seat with headrest), but there was no statistically significant difference across seating conditions ( $p>0.05$, Friedman).

\subsection{Effect of seat inclination on discomfort}

After correcting for multiple comparisons, there were no statistically significant effects of seat inclination on magnitude estimates of discomfort at any of the six magnitudes of vibration ( $p \geq 0.192$, Friedman).

Vibration acceleration magnitudes corresponding to subjective magnitudes, $\psi$, of 50, 63, $80,100,125,160,200,250$, and 315 , were calculated for each subject and each seating condition using the rate of growth of discomfort, $n$, and constant, $k$, for each individual. As expected from the absence of a dependence of the rate of growth of discomfort on seating condition, the median vibration magnitudes corresponding to these six subjective magnitudes increased similarly with increasing vibration magnitude for each seating condition (Figure 5). The interquartile range of normalised responses also varied little across the six seating conditions (Figure 5).

\section{Figure 5 about here}

\subsection{Location of discomfort}

When seated either upright or nearly upright with no headrest, subjects felt most discomfort in their buttocks near the point of contact with the seat pan (area 6, Figure 6), but with the seat reclined to $45^{\circ}$ and $60^{\circ}$ most discomfort was experienced in the middle-back and the upper-back (areas 3 and 4, Figure 6). The percentage of subjects reporting discomfort in the lower-back (area 5, Figure 6), was similar in each of the four reclined seating conditions. 
Figure 6 about here

\section{Discussion}

\subsection{Predicting the influence of seat inclination on vibration discomfort}

With vertical acceleration, $a$, as a seat is reclined the components of acceleration in the $x$-axis at the seat pan and the backrest (i.e., $a \cdot \sin \theta)$ increase and the components of acceleration in the $z$-axis (i.e., $a \cdot \cos \theta$ ) decrease. With $1-\mathrm{Hz}$ vertical vibration, the frequency weightings in BS 6841:1987 indicate that if a seat is reclined by more than $22^{\circ}$ the $x$-axis component of acceleration at the seat pan will cause more discomfort than the $z$-axis component at the seat pan. With $16-\mathrm{Hz}$ vertical vibration, the $z$-axis acceleration at the seat pan is the dominant component for all seat inclinations up to $63^{\circ}$ (Figure 7). When using the weightings for comfort in ISO 2631-1:1997 the $x$-axis component of a $1-\mathrm{Hz}$ vertical motion is predicted to be dominant at the seat pan when it is inclined by $27^{\circ}$, but the angle is reduced to $20^{\circ}$ when using the 1.4 multiplying factor for fore-and-aft vibration suggested in ISO 2631-1 (1997) for evaluating vibration and shock with respect to health.

The weightings in BS 6841 (1987) show not only how the overall ride value varies with seat inclination but also the relative importance of the two components of motion at the seat pan and the two components at the seat back (Figure 7). At all frequencies, as the seat reclines the $z$-axis components reduce (to zero when fully reclined at $90^{\circ}$ ) while the $x$-axis components increase. At $1-\mathrm{Hz}$, the $x$-axis component begins to dominate at the seat pan when the seat is inclined by more than about $21^{\circ}$ and dominate at the seat back when it is inclined by more than about 27 degrees. Although this seems reasonable for $x$-axis vibration of the backrest it seems unreasonable for $x$-axis vibration at the seat pan, which becomes the greatest of the four component ride values when the seat is 
reclined by more than about 22 degrees. This anomaly reduces as the frequency increases: $x$-axis backrest vibration becomes more severe than $x$-axis seat pan vibration when the frequency is greater than about $2.5 \mathrm{~Hz}$, and $z$-axis seat pan vibration remains more important than both of the two $x$-axis components up to greater inclinations. The anomalous importance of the $x$-axis component at the seat for low frequency vibration arising from vertical vibration of an inclined seat pan seems to have been overlooked previously. The anomaly is greater when using the 1.4 multiplying factor for $x$-axis vibration as suggested in ISO 2631-1 (1997) for the evaluation of vibration with respect to health. It seems likely that the $x$-axis vibration measured on inclined seats will often be influenced by the vertical vibration, even though there are no experimental data suggesting how this component should be evaluated.

\section{Figure 7 about here}

\subsection{Predicting the influence of backrest inclination on vibration discomfort}

As a seat back reclines while the seat pan remains horizontal, the $z$-axis component of vertical acceleration at the back decreases, the $x$-axis component of acceleration at the back increases, the $z$-axis component of acceleration at the seat pan remains constant, and there is no $x$-axis component at the seat pan. At $1-\mathrm{Hz}$, the $x$-axis component at the back dominates when the seat is inclined by more than $30^{\circ}$. At $4 \mathrm{~Hz}$ and $16 \mathrm{~Hz}$, the $z-$ axis acceleration at the seat surface is the dominant component for all backrest inclinations (Figure 7). The overall ride value increases with increasing backrest inclination at all frequencies, although the predicted influence of backrest inclination reduces as the frequency of vibration increases (Figure 8).

Figure 8 about here 


\subsubsection{Comparison with previous studies}

For subjects sitting on a horizontal seat pan with rigid flat backrest, when the backrest was reclined to 30 degrees their discomfort only increased at frequencies greater than 8 $\mathrm{Hz}$, and there was no further increase in discomfort as the backrest reclined to the horizontal (Basri and Griffin 2013). This differs from the standards that suggest the greatest increase will occur at the lower frequencies (Figure 7). When supported by a horizontal seat pan that vibrated vertically and a stationary backrest, there was reduced discomfort at all frequencies from 2.5 to $25 \mathrm{~Hz}$ as the stationary backrest reclined from vertical to horizontal, whereas the standards predict no change in discomfort (Basri and Griffin 2012). The effect may be associated with less weight being supported on the seat as more weight is supported on the backrest. Such an effect probably occurred in the present experiment, resulting in the contribution of seat vibration (in both the $z$-axis and $x$-axis) to overall discomfort being over-estimated by the standards, an anomaly most noticeable at low frequencies (Figure 7).

With $x$-axis backrest vibration there does not seem to be a systematic effect of the increased weight of the body supported on the backrest, although the frequency weightings differ between an upright backrest and a backrest reclined between 30 and 90 degrees (Basri and Griffin 2011a). With $z$-axis backrest vibration there was also no systematic effect of backrest inclination and a similar frequency-dependence was found with all backrest inclinations from 0 to 90 degrees (Basri and Griffin 2011b).

\subsection{Predicting the influence of seat inclination on boat motion discomfort}

Overall ride values for the vertical boat motion in the current study calculated from the $x$-axis and $z$-axis components at the seat pan and the seat back according to BS 6841:1987 show an $82 \%$ increase as the seat was reclined from $0^{\circ}$ to $90^{\circ}$ (Figure 9). 
This predicts an increase in discomfort inconsistent with the experimental findings that showed no significant change in discomfort with this change in seat inclination. The same analysis using vibration dose values predicts a $50 \%$ increase due to reclining the seat, also inconsistent with the experimental findings.

\section{Figure 9 about here}

As a seat pan is reclined, the standards do not seem to apply appropriate weight to the $x$ axis component of seat pan vibration arising from vertical vibration. As a seat back is reclined, the standards do not reduce the importance of $z$-axis seat pan vibration as more weight of the body is supported on the seat back. Revised weightings would be needed for the seat pan to account for the effects of seat pan inclination (on discomfort caused by $x$-axis vibration) and seat back inclination (on discomfort caused by $z$-axis seat pan vibration) before a reasonable prediction can be made. The experimental findings of the present study may therefore not apply to motions with other frequencies of vibration and other types of shock.

\section{Conclusions}

When exposed to simulations of recorded fast boat motion, there was little or no change in the discomfort of healthy subjects when sitting upright in a seat with or without backrest or sitting in the same seat with the seat pan and backrest reclined to $15^{\circ}, 30^{\circ}$, $45^{\circ}$, or $60^{\circ}$. The findings suggest that reclined seats in fast boats may be acceptable in respect of comfort. The findings are inconsistent with the predictions of current standards. It seems likely that the standards do not provide suitable weightings for the vibration at the seat or backrest when a seat is reclined.

\section{Acknowledgements}

Research funded by DES SE Sea-ShipDes, MOD Abbey Wood, Bristol. 


\section{References}

Basri, B. and Griffin, M.J. 2011a. "The vibration of inclined backrests: perception and discomfort of vibration applied normal to the back in the x-axis of the body." Journal of Sound and Vibration, 330 (18-19), 4646-4659.

Basri, B. and Griffin, M.J. 2011b. "The vibration of inclined backrests: perception and discomfort of vibration applied parallel to the back in the z-axis of the body." Ergonomics 54:12 1214-1227.

Basri, B. and Griffin, M.J. 2012. "Equivalent comfort contours for vertical seat vibration: Effect of vibration magnitude and backrest inclination." Ergonomics 55 (8), 909-922.

Basri, B. and Griffin, M.J. 2013. "Predicting discomfort from whole-body vertical vibration when sitting with an inclined backrest." Applied Ergonomics 44, (3), 423-434.

British Standards Institution. 1987. "Measurement and evaluation of human exposure to wholebody mechanical vibration and repeated shock." BS 6841. London: British Standards Institution.

Howarth, H.V.C. and Griffin, M.J. 2012. "Predicting the influence of seat and backrest inclination on the discomfort of whole-body vertical vibration on high-speed craft." Paper presented at the 47th UK Conference on Human Responses to Vibration, held at ISVR, University of Southampton, England, Sept 2012.

Huang, Y. and Griffin, M.J. 2014. "Comparison of absolute magnitude estimation and relative magnitude estimation for judging the subjective intensity of noise and vibration." Applied Acoustics 77, 82-88.

International Organization for Standardization. 1997. "Mechanical vibration and shock evaluation of human exposure to whole-body vibration - Part 1: General requirements." ISO 2631-1. International Organization for Standardization, Geneva.

International Organization for Standardization. 2004. "Mechanical vibration and shock -Evaluation of human exposure to whole-body vibration -- Part 5: Method for evaluation of vibration containing multiple shocks." ISO 2631-5. International Organization for Standardization, Geneva.

Kitazaki, S. and Griffin, M.J. 1998. "Resonance behaviour of seated human body and effects of posture." Journal of Biomechanics 31, 143-149.

Mansfield, N.J., and Griffin, M.J. 2002. "Effects of posture and vibration magnitude on apparent mass and pelvis rotation during exposure to whole-body vertical vibration." Journal of Sound and Vibration, 253(1), 93-107.

Messenger, A.J., and Griffin, M.J. 1990 "Lumbar support in seating: Effects on erector spinae muscle activity, posture and discomfort." In: Contemporary Ergonomics 1990, Editor: E.J. Lovesey. Published: Taylor and Francis, London, ISBN: 0-85066-851-4. Proceedings of the Ergonomics Society's Annual Conference 'Ergonomics - Setting Standards for the '90's', 293298.

Paddan, G.S. and Griffin, M.J. 1988. "The transmission of translational seat vibration to the head - I. Vertical seat vibration.” Journal of Biomechanics, 21(3), 191-197.

Paddan, G.S., and Griffin, M.J. 1998, "A review of the transmission of translational seat vibration to the head." Journal of Sound and Vibration, 215(4), 863-882. 
Paddan. G.S., Mansfield, N.J., Arrowsmith, C.I., Rimell, A.N., King, S.K. and Holmes S.R. 2012, "The influence of seat backrest angle on perceived discomfort during exposure to vertical whole-body vibration." Ergonomics, 55(8), 923-936.

Stevens, S.S. 1975. Psychophysics: introduction to its perceptual, neural, and social prospects. New York: J Wiley \& Sons Inc.

Toward, M.G.R. and Griffin, M.J. 2009. "Apparent mass of the human body in the vertical direction: Effect of seat backrest." Journal of Sound and Vibration 327, 657-669.

Wang, W. Rakheja, S. and Boileau, P.E. 2004 "Effects of sitting postures on biodynamic response of seated occupant under vertical vibration", International Journal of Industrial Ergonomics 34, 289-306. 


\section{Figure captions}

Figure 1. Overall ride values calculated from $x$-axis and $z$-axis components of vibration at the seat pan and seat back with $10 \mathrm{~ms}^{-2}$ r.m.s. vertical acceleration when seat pan and seat backrest are both reclined from $0^{\circ}$ to $90^{\circ}$ according to BS 6841:1987 (from Howarth and Griffin 2012).

Figure 2. Time histories and power spectral densities of acceleration (frequency resolution $0.2 \mathrm{~Hz}, 16$ degrees of freedom) of the fast-boat motion recorded on the simulator platform at the greatest magnitude.

Figure 3. Subject sitting on the motion simulator in the six seating conditions: (i) upright, no backrest, no headrest; (ii) $15^{\circ}$, no headrest; (iii) $30^{\circ}$, no headrest; (iv) $30^{\circ}$ with headrest; (v) $45^{\circ}$ with headrest; (vi) $60^{\circ}$ with headrest.

Figure 4. Median normalised magnitude estimates of discomfort for the six seating conditions.

Figure 5. Vibration acceleration magnitudes corresponding to magnitude estimates from $\psi=50$ to $\psi=315$ (top) and magnitude estimates of discomfort caused by the greatest magnitude of vibration with box plot showing $25^{\text {th }}, 50^{\text {th }}$, and $75^{\text {th }}$ percentiles and whiskers showing minima and maxima (bottom): (i) upright, no backrest, no headrest; (ii) $15^{\circ}$, no headrest; (iii) $30^{\circ}$, no headrest; (iv) $30^{\circ}$ with headrest; (v) $45^{\circ}$ with headrest; (vi) $60^{\circ}$ with headrest.

Figure 6. Principal locations of discomfort in the body caused by the greatest magnitude of vibration: (i) upright, no backrest, no headrest; (ii) $15^{\circ}$, no headrest; (iii) $30^{\circ}$, no headrest; (iv) $30^{\circ}$ with headrest; (v) $45^{\circ}$ with headrest; (vi) $60^{\circ}$ with headrest.

Figure 7 Frequency-weighted $x$-axis and $z$-axis components at the seat pan and seat back and overall ride values with $10 \mathrm{~ms}^{-2}$ r.m.s. vertical acceleration when the seat pan and seat backrest are reclined (left) and when only the backrest is reclined (right) from $0^{\circ}$ to $90^{\circ}$ according to BS $6841: 1987$.

Figure 8. Overall ride values calculated from $x$-axis and $z$-axis components of vibration at the seat pan and seat back with $10 \mathrm{~ms}^{-2}$ r.m.s. vertical acceleration when only the backrest is reclined from $0^{\circ}$ to $90^{\circ}$ according to BS 6841:1987.

Figure 9. Frequency-weighted $x$-axis and $z$-axis components at the seat pan and seat back and overall ride values (top) and overall vibration dose values (bottom) with vertical fast-boat motion at the greatest magnitude when both the seat pan and seat backrest are reclined from $0^{\circ}$ to $90^{\circ}$ according to BS 6841:1987.

\section{Table captions}

Table 1. Guidance in BS 6841 (1987) and ISO 2631-1 (1997) for the application of frequency weightings and multiplying factors for evaluation of whole-body vibration with respect to comfort.

Table 2. Ranges of frequency-weighted absolute peak acceleration, overall r.m.s. acceleration, and VDV of the six magnitudes of 20-s motion recorded on the simulator platform. 


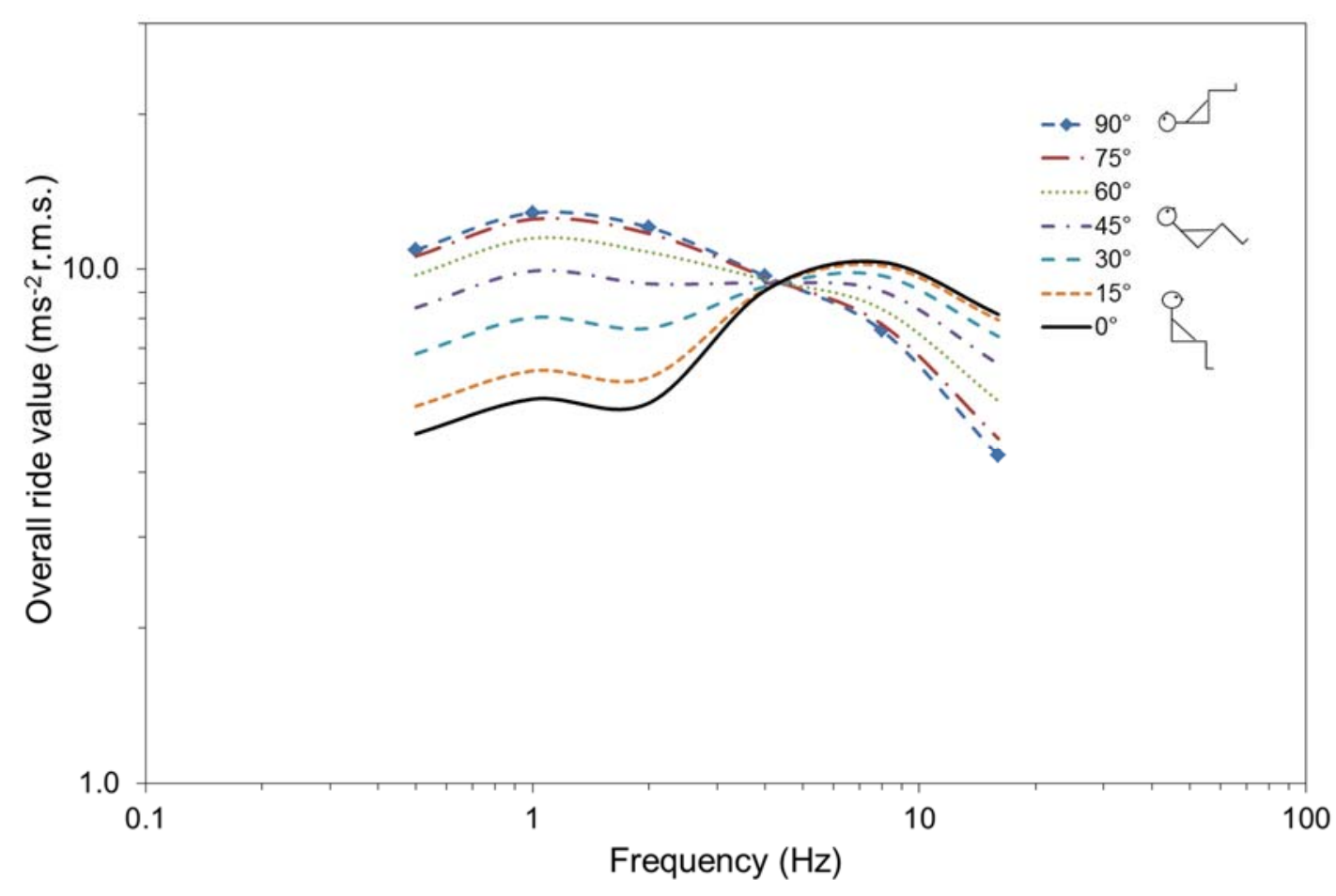

Figure 1 Overall ride values calculated from $x$-axis and $z$-axis components of vibration at the seat pan and seat back with $10 \mathrm{~ms}^{-2}$ r.m.s. vertical acceleration when seat pan and seat backrest are both reclined from $0^{\circ}$ to $90^{\circ}$ according to BS 6841:1987 (from Howarth and Griffin 2012). 


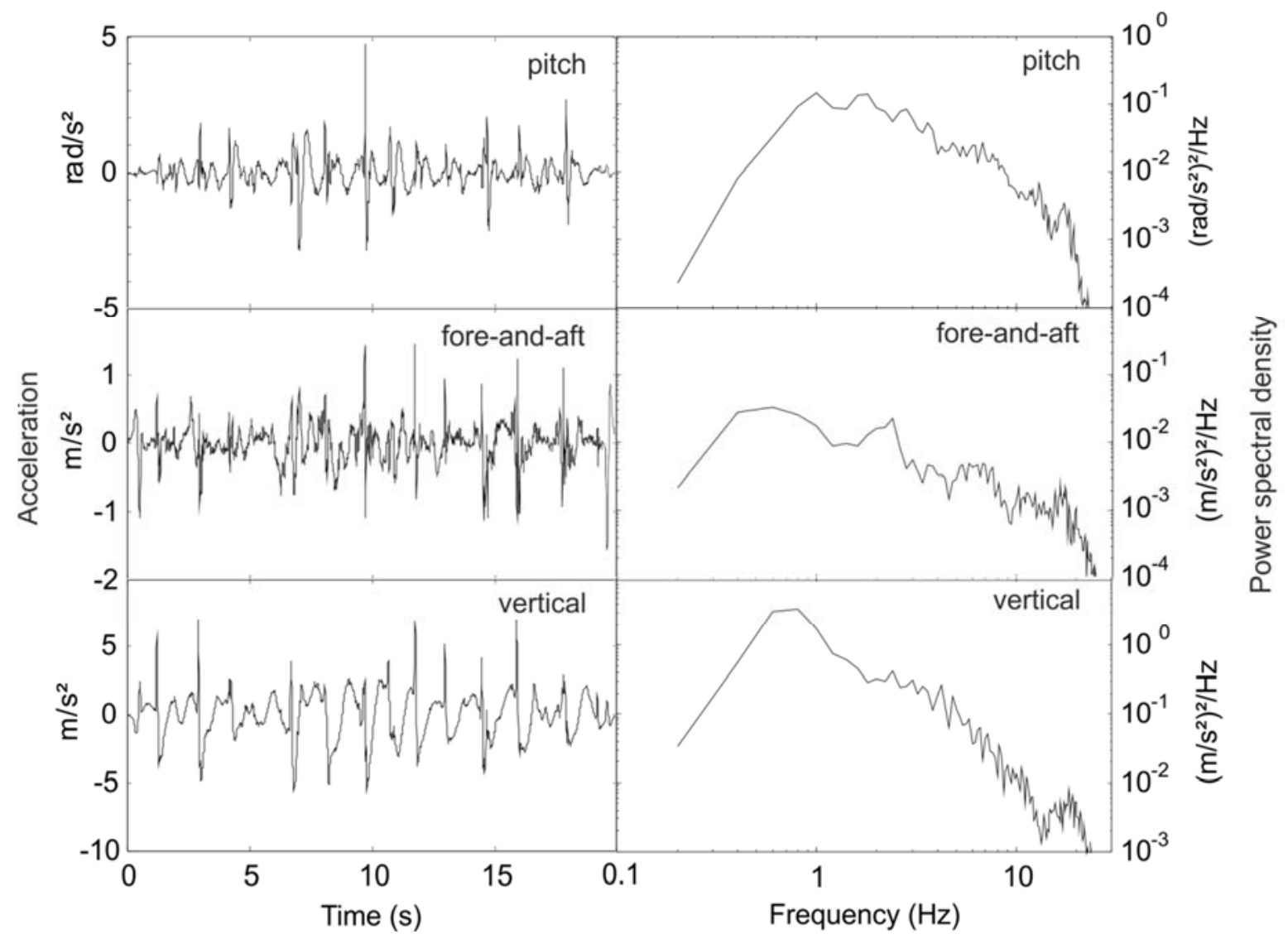

Figure 2 Time histories and power spectral densities of acceleration (frequency resolution $0.2 \mathrm{~Hz}, 16$ degrees of freedom) of the fast-boat motion recorded on the simulator platform at the greatest magnitude. 

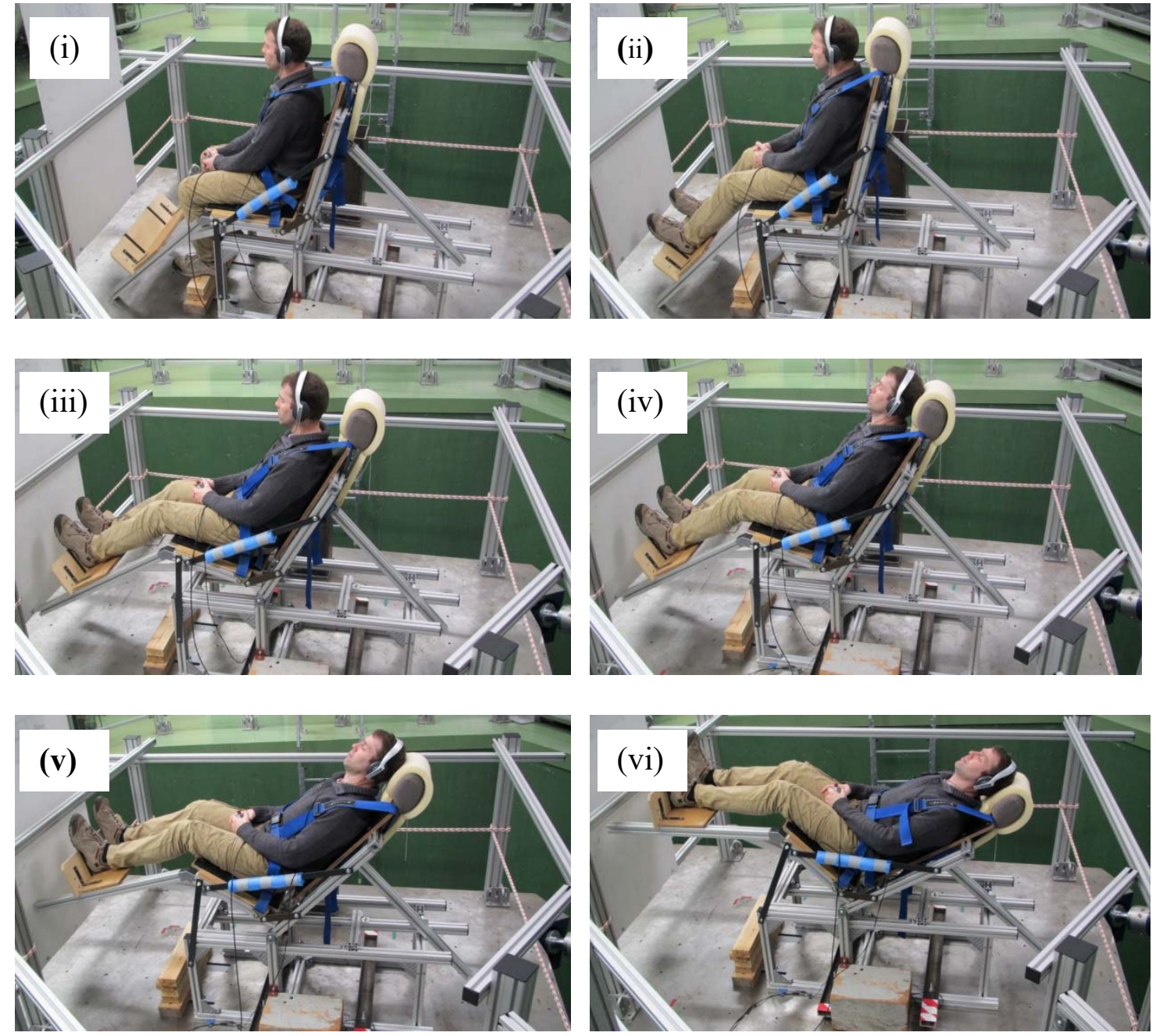

Figure 3. Subject sitting on the motion simulator in the six seating conditions: (i) upright, no backrest, no headrest; (ii) $15^{\circ}$, no headrest; (iii) $30^{\circ}$, no headrest; (iv) $30^{\circ}$ with headrest; (v) $45^{\circ}$ with headrest; (vi) $60^{\circ}$ with headrest. 


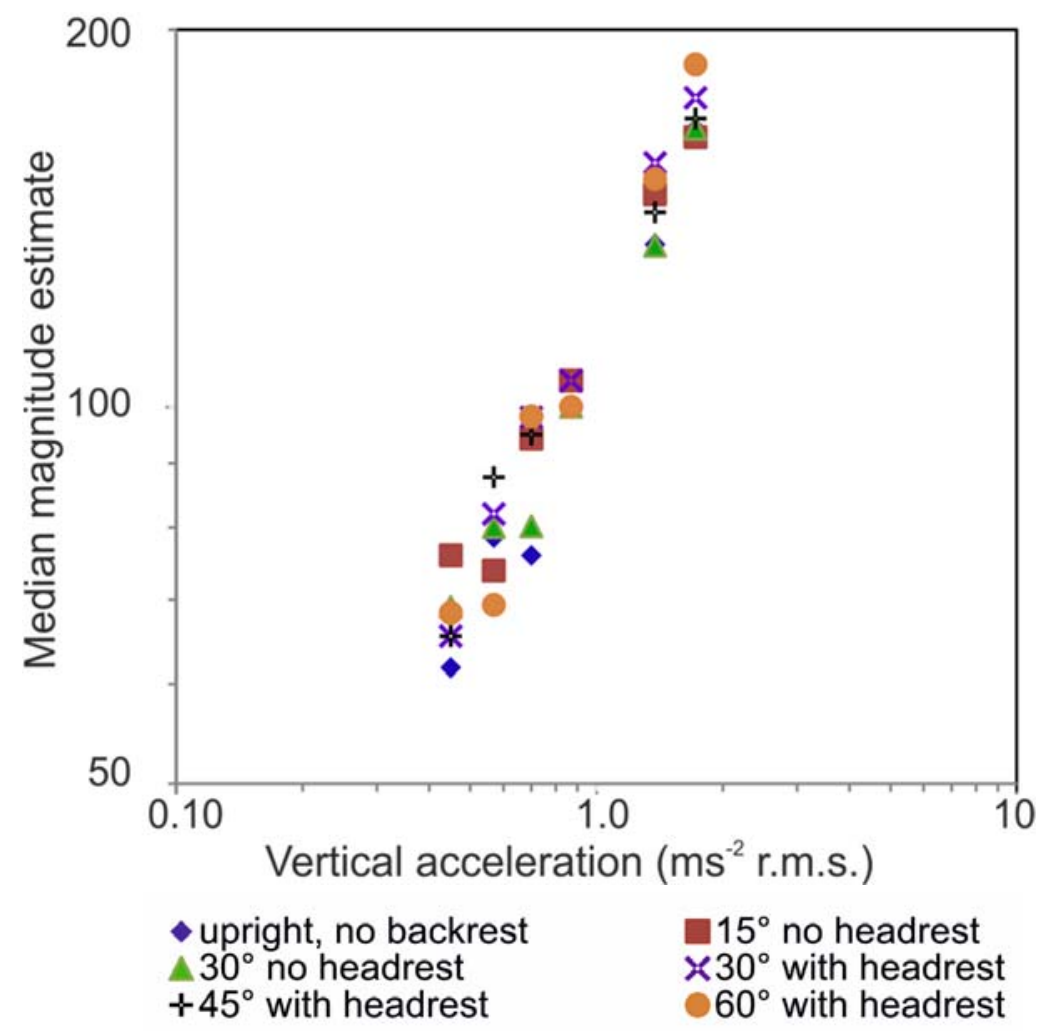

Figure 4. Median normalised magnitude estimates of discomfort for the six seating conditions. 


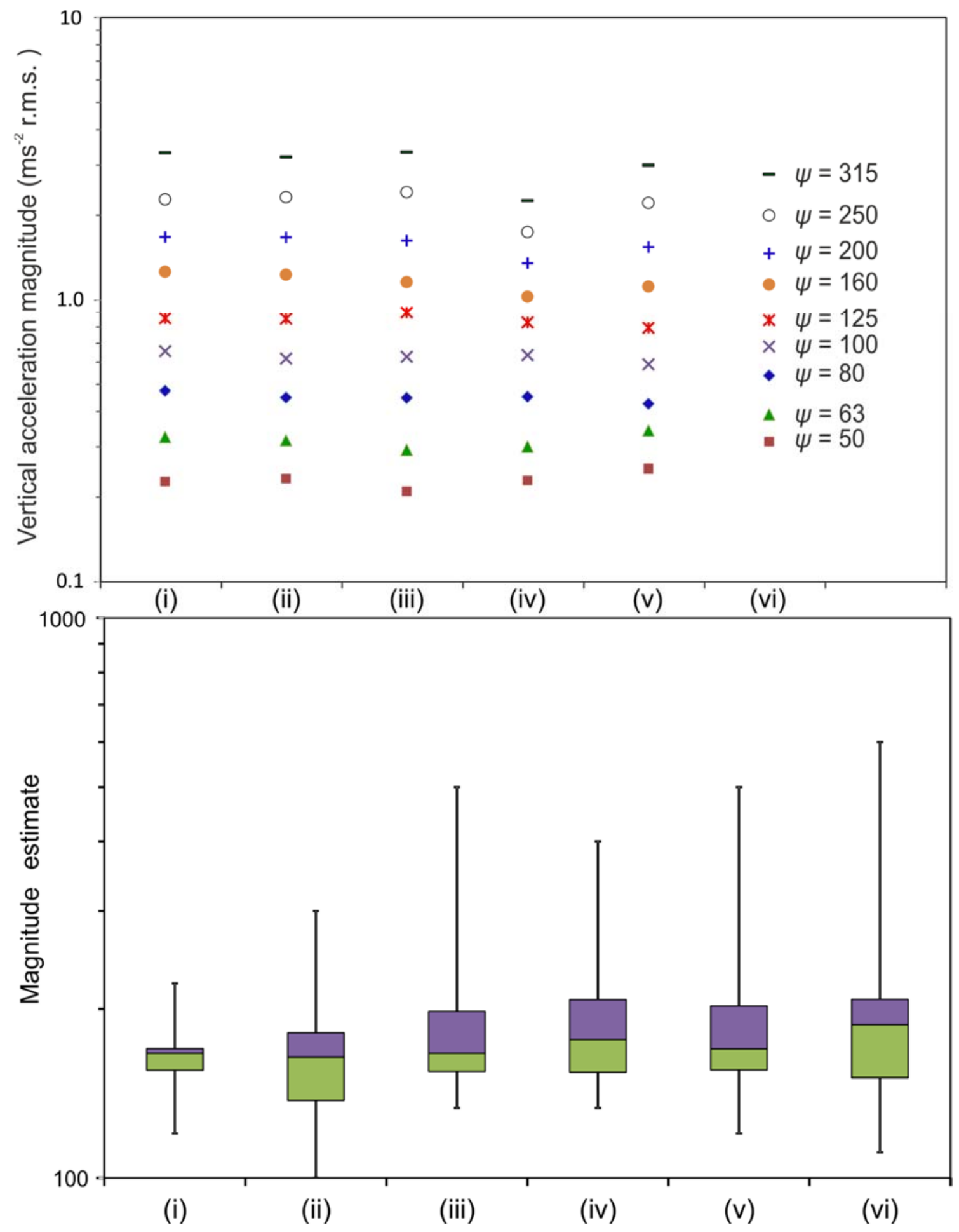

Figure 5 Vibration acceleration magnitudes corresponding to magnitude estimates from $\psi=50$ to $\psi=315$ (top) and magnitude estimates of discomfort caused by the greatest magnitude of vibration with box plot showing $25^{\text {th }}, 50^{\text {th }}$, and $75^{\text {th }}$ percentiles and whiskers showing minima and maxima (bottom): (i) upright, no backrest, no headrest; (ii) $15^{\circ}$, no headrest; (iii) $30^{\circ}$, no headrest; (iv) $30^{\circ}$ with headrest; (v) $45^{\circ}$ with headrest; (vi) $60^{\circ}$ with headrest. 


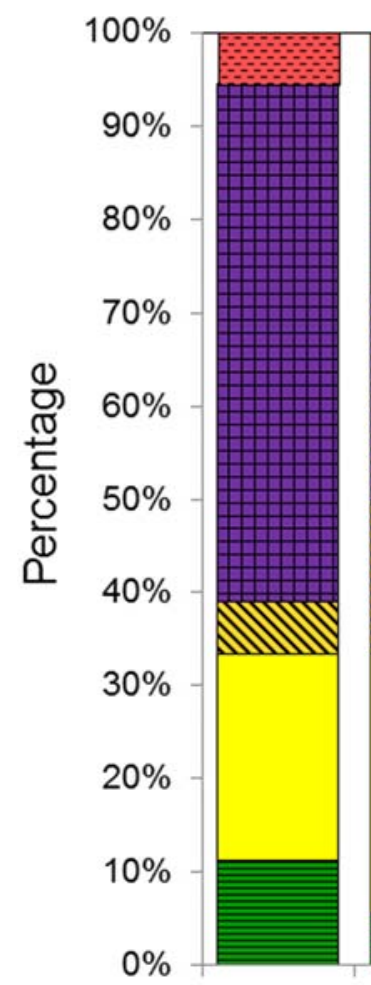

(i)

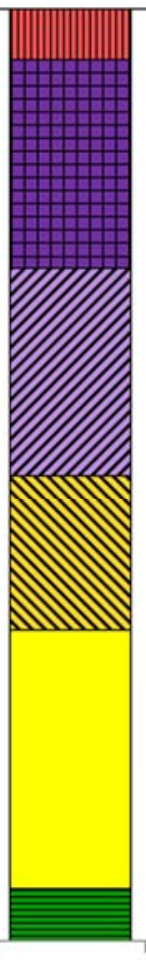

(ii)

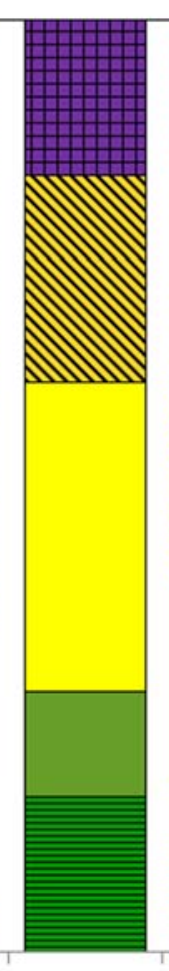

(iii)

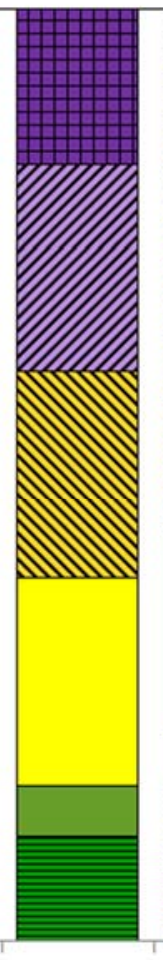

(iv)

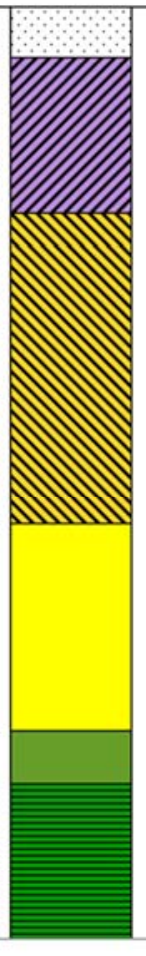

( $v)$

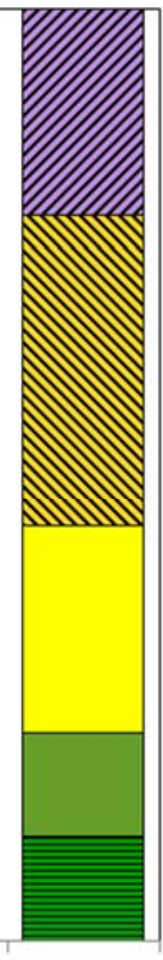

(vi)

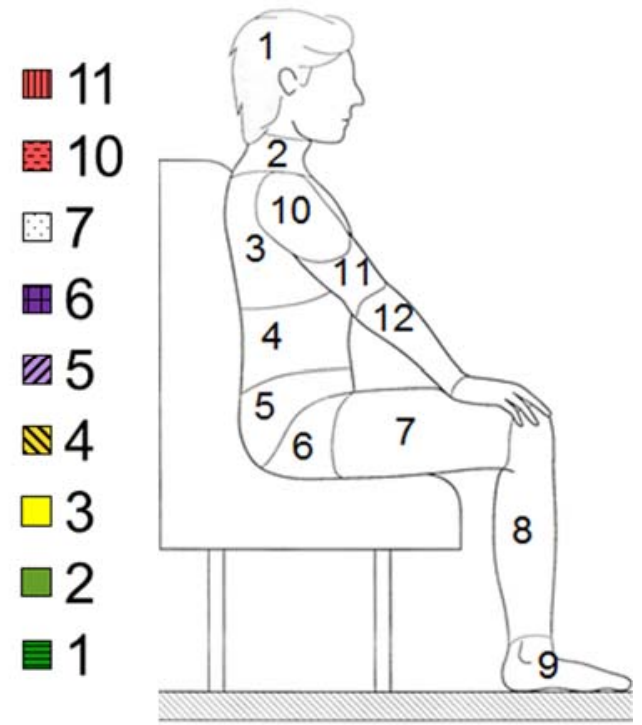

Figure 6 Principal locations of discomfort in the body caused by the greatest magnitude of vibration: (i) upright, no backrest, no headrest; (ii) $15^{\circ}$, no headrest; (iii) $30^{\circ}$, no headrest; (iv) $30^{\circ}$ with headrest; (v) $45^{\circ}$ with headrest; (vi) $60^{\circ}$ with headrest. 


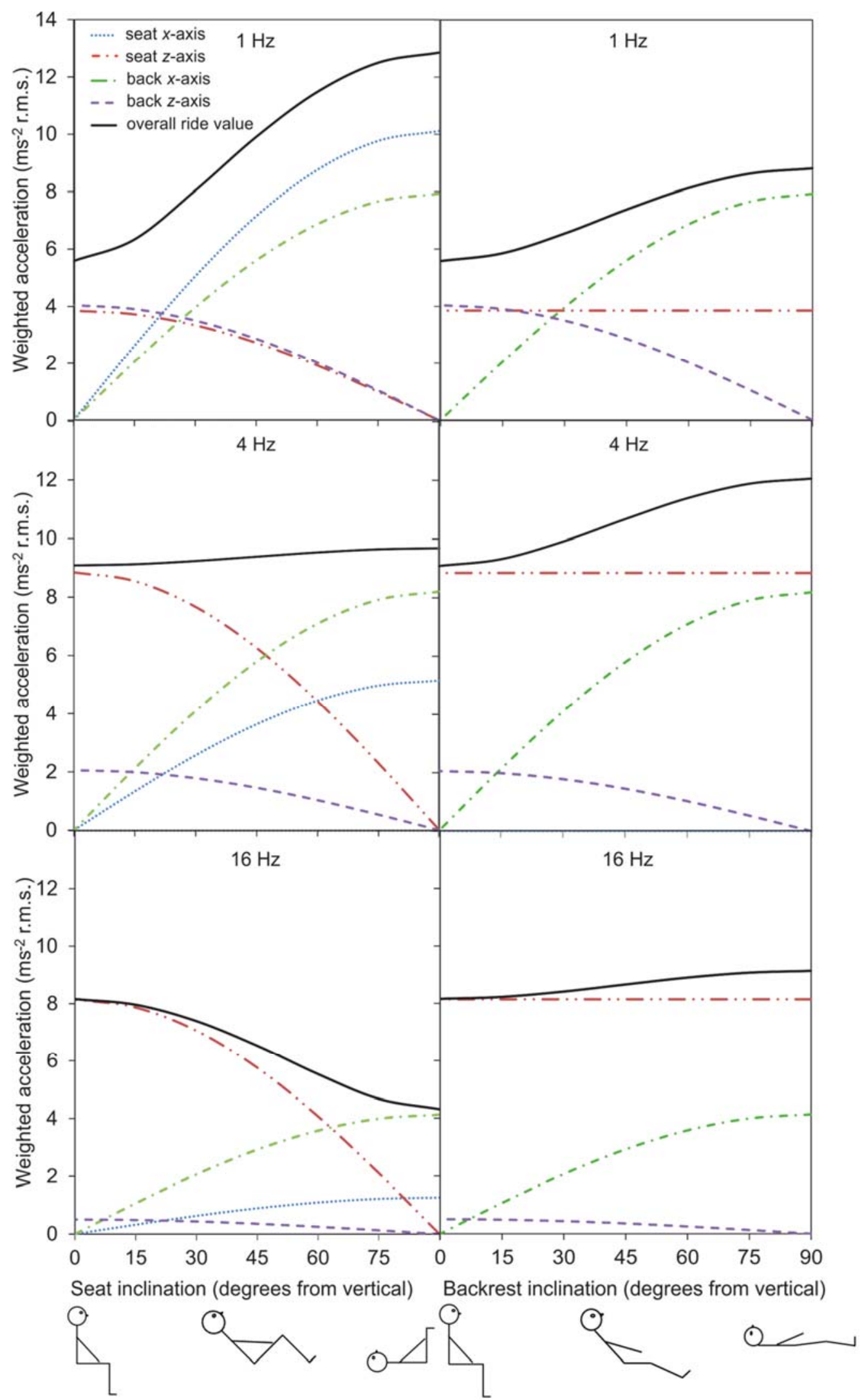

Figure 7 Frequency-weighted $x$-axis and $z$-axis components at the seat pan and seat back and overall ride values with $10 \mathrm{~ms}^{-2}$ r.m.s. vertical acceleration when the seat pan and seat backrest are reclined (left) and when only the backrest is reclined (right) from $0^{\circ}$ to $90^{\circ}$ according to BS 6841:1987. 


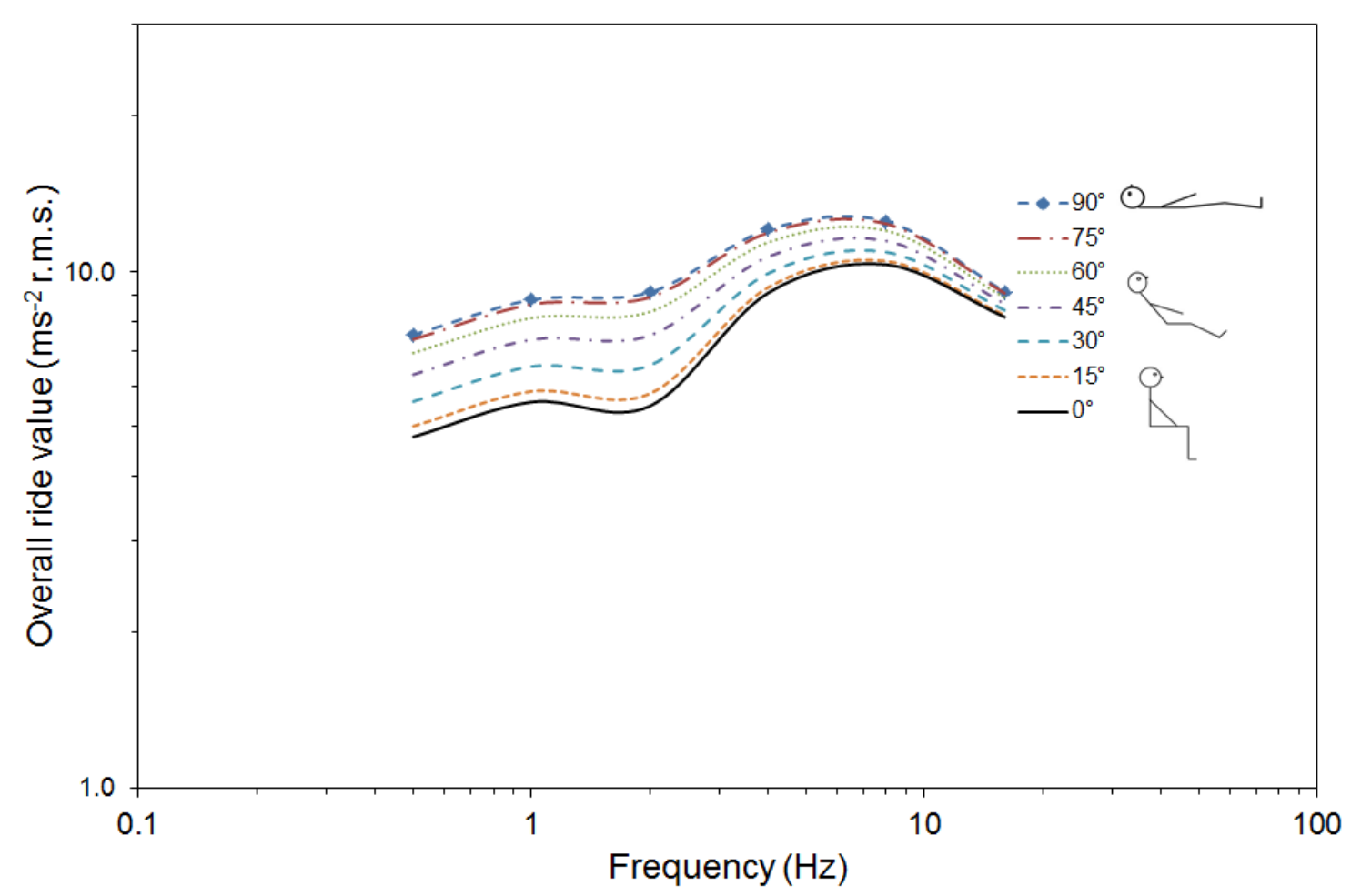

Figure 8. Overall ride values calculated from $x$-axis and $z$-axis components of vibration at the seat pan and seat back with $10 \mathrm{~ms}^{-2}$ r.m.s. vertical acceleration when only the backrest is reclined from $0^{\circ}$ to $90^{\circ}$ according to BS 6841:1987. 


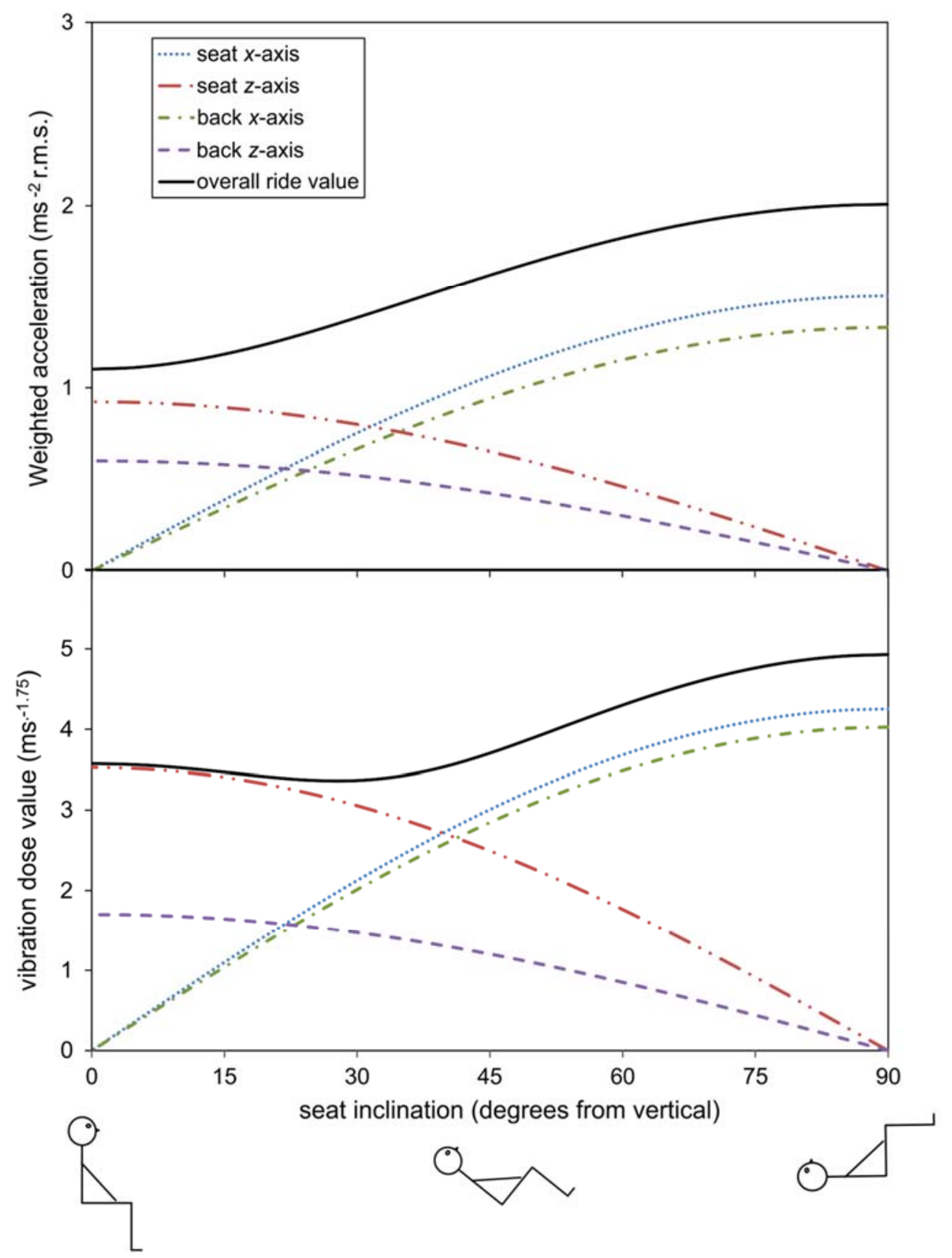

Figure 9. Frequency-weighted $x$-axis and $z$-axis components at the seat pan and seat back and overall ride values (top) and overall vibration dose values (bottom) with vertical fast-boat motion at the greatest magnitude when both the seat pan and seat backrest are reclined from $0^{\circ}$ to $90^{\circ}$ according to BS 6841:1987. 
Table 1. Guidance in BS 6841 (1987) and ISO 2631-1 (1997) for the application of frequency weightings and multiplying factors for evaluation of whole-body vibration with respect to comfort.

\begin{tabular}{|l|cc|cc|}
\hline \multirow{2}{*}{ Measurement axis } & \multicolumn{2}{|c|}{ BS 6841 (1987) } & \multicolumn{2}{c|}{ ISO 2631-1 (1997) } \\
& $\begin{array}{c}\text { Frequency } \\
\text { weighting }\end{array}$ & $\begin{array}{c}\text { Multiplying } \\
\text { factor, } k\end{array}$ & $\begin{array}{c}\text { Frequency } \\
\text { weighting }\end{array}$ & $\begin{array}{c}\text { Multiplying } \\
\text { factor, } k\end{array}$ \\
\hline$x$-axis on seat & $W_{\mathrm{d}}$ & 1.0 & $W_{\mathrm{d}}$ & 1.0 \\
$y$-axis on seat & $W_{\mathrm{d}}$ & 1.0 & $W_{\mathrm{d}}$ & 1.0 \\
z-axis on seat & $W_{\mathrm{b}}$ & 1.0 & $W_{\mathrm{k}}$ & 1.0 \\
$x$-axis on seat back & $W_{\mathrm{c}}$ & 0.8 & $W_{\mathrm{c}}$ & 0.8 \\
$y$-axis on seat back & $W_{\mathrm{d}}$ & 0.5 & $W_{\mathrm{d}}$ & 0.5 \\
$z$-axis on seat back & $W_{\mathrm{d}}$ & 0.4 & $W_{\mathrm{d}}$ & 0.4 \\
$x$-axis feet & $W_{\mathrm{b}}$ & 0.25 & $W_{\mathrm{k}}$ & 0.25 \\
$y$-axis feet & $W_{\mathrm{b}}$ & 0.25 & $W_{\mathrm{k}}$ & 0.25 \\
$z$-axis feet & $W_{\mathrm{b}}$ & 0.4 & $W_{\mathrm{k}}$ & 0.4 \\
$r_{\mathrm{x}}$-axis seat & $W_{\mathrm{e}}$ & 0.63 & $W_{\mathrm{e}}$ & 0.63 \\
$r_{\mathrm{y}}$-axis seat & $W_{\mathrm{e}}$ & 0.4 & $W_{\mathrm{e}}$ & 0.4 \\
$r_{\mathrm{z}}$-axis seat & $W_{\mathrm{e}}$ & 0.2 & $W_{\mathrm{e}}$ & 0.2 \\
\hline
\end{tabular}


Table 2 Ranges of frequency-weighted absolute peak acceleration, overall r.m.s. acceleration, and VDV of the six magnitudes of 20 -s motion recorded on the simulator platform.

\begin{tabular}{|c|c|c|c|}
\hline \multirow{2}{*}{ Axis } & \multicolumn{2}{|c|}{$\begin{array}{c}\text { Frequency-weighted } \\
\text { acceleration }\end{array}$} & \multirow{2}{*}{ VDV } \\
\cline { 2 - 3 } & Peak & Overall r.m.s. & \\
\hline Vertical $\left(W_{\mathrm{b}}\right)$ & $1.65-5.21 \mathrm{~ms}^{-2}$ & $0.26-0.92 \mathrm{~ms}^{-2}$ & $1.05-3.53 \mathrm{~ms}^{-1.75}$ \\
\hline Fore-and-aft $\left(W_{\mathrm{d}}\right)$ & $0.22-0.79 \mathrm{~ms}^{-2}$ & $0.05-0.20 \mathrm{~ms}^{-2}$ & $0.16-0.62 \mathrm{~ms}^{-1.75}$ \\
\hline Pitch $\left(W_{\mathrm{e}}\right)$ & $0.31-1.30 \mathrm{rads}^{-2}$ & $0.08-0.30 \mathrm{rads}^{-2}$ & $0.24-0.95 \mathrm{rads}^{-1.75}$ \\
\hline
\end{tabular}

